\title{
Kinematic modeling and validation of a 6-DOF industrial robot
}

\section{Guo-Qing Sun ${ }^{1, a}$ and Guan-Bin Gao ${ }^{1, b}$}

${ }^{1}$ Faculty of Mechanical and Electrical Engineering, Kunming University of Science and Technology, Kunming 650500, China.

aguoqingdj@163.com, Corresponding author: bgbgao@163.com

Keywords: Industrial robot, Kinematic model, Graphic simulation, Transformation.

\begin{abstract}
The kinematic model of industrial robots is the transformation between the working space and the space of joints. After analysis of the kinematic methods in the area of linkage mechanisms, the kinematic model was established according to DH method for a 6-DOF industrial robot. To verify the kinematic model, the graphic simulation of the industrial robot was established with Robotics Toolbox in Matlab. The calculation result of the kinematic model and the simulation are consistent. Experiments were carried out to verify the kinematic model, which shows that the kinematic model is correct.
\end{abstract}

\section{Introduction}

Industrial robots has been widely used in industrial production, the main application of which are assembly, welding, painting, etc. The kinematic modeling is very important for robots, and it is also the basis of calibration, trajectory planning and motion control. Kinematic through the determination of structural parameters the position and orientation of the robot's end-effector can be determined according to the kinematic modeling. Furthermore, the relationship between the position of the end-effector and the displacement of the joint angle can be described by the kinematic model.

This paper takes a 6-DOF serial robot as the research object, the structure of which is shown in Fig. 1. The kinematic model of the robot was established according to DH method. And the kinematic model was verified by the simulation result.

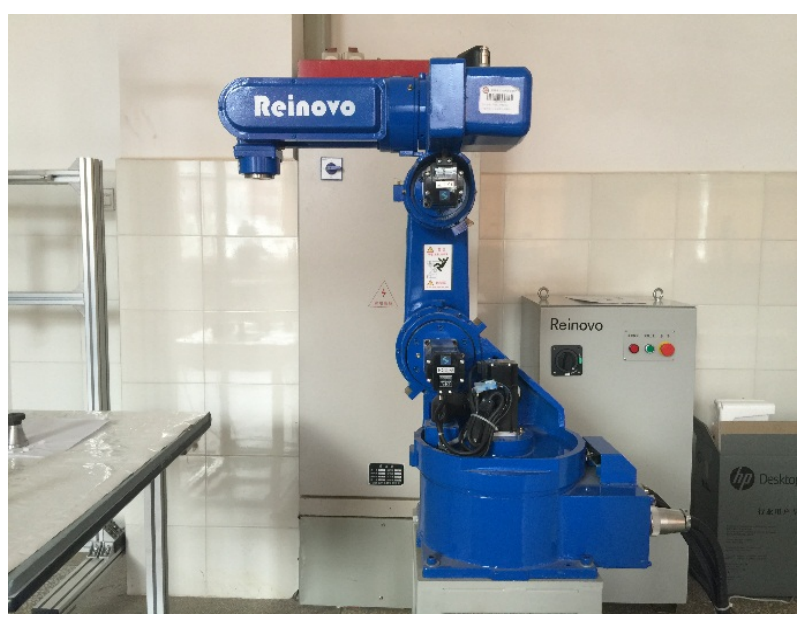

Fig. 1 The 6-DOF industrial robot

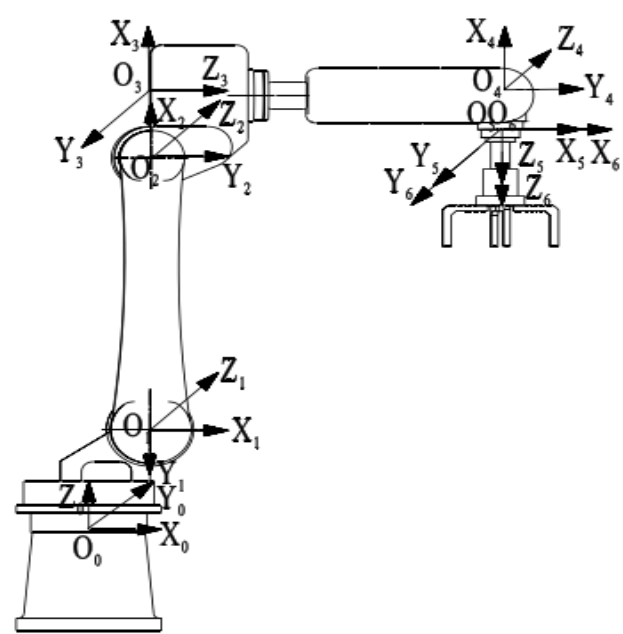

Fig. 2 The coordinate systems of the robot

The most classic and basic robot kinematic modeling method is Denavit and Hartenberg method (referred to as DH), which was proposed by Denavit and Hartenberg. According to certain rules, the joint coordinate system is fixed on the corresponding linkage of the robot. The relationship of the adjacent two linkages can be described through a homogeneous transformation matrix. However, there are also some disadvantages in DH model, e.g. the singularity problem when two adjacent linkages are parallel or nearly parallel. In order to overcome the singular problem many scholars proposed improved methods of DH model or new models. 


\section{Kinematic analysis of the 6-DOF industrial robot}

The basic steps of DH method are as follows:

(1) Determine the $z$ axis, $z_{i}$ axis is along the axial joints $i+1$;(The direction of the arrow can be arbitrarily chosen, but usually every point in the same direction parallel to the axis of the $z$ axis.)

(2) Determining the origin $o$, the $o_{i}$ is on the common perpendicular of $z_{i}$ and $z_{i-1}$;

(3) Determining the $x$ axis, the $x_{i}$ axis is along the direction of the common perpendicular of $z_{i}$ and $z_{i-1}$ and leaving the $z_{i-1}$ axis;

(4) Determining the $y$ axis, according to the right-hand rule. That is $y_{i}=z_{i} \times x_{i}$.

According to the above steps, the coordinate systems of each joint of are established. The 4 parameters for describing the relative position between adjacent coordinates can be determined:

(1) $d_{i}$ is the distance between the axis of $x_{i-1}$ and $x_{i}$, along $z_{i}$ direction is positive;

(2) $a_{i}$ is the distance between the axis of $z_{i-1}$ and $z_{i}$, along $x_{i-1}$ direction is positive;

(3) $\alpha_{i}$ is the angle between the axis of $z_{i-1}$ and $z_{i}$, around $x_{i-1}$ counter-clockwise;

(4) $\theta_{i}$ is $x_{i-1}$ the angle between the axis of $x_{i}$ and $x_{i-1}$, around $z_{i}$ counter-clockwise;

Following the above steps, we can get coordinate systems and the parameters of the robot shown as Fig. 2 and Table 1.

The parameters of the robot are shown in Table 1:

Table 1 Parameters of the industrial robot

\begin{tabular}{ccccc}
\hline Joint No. & $\alpha_{i-1}\left({ }^{\circ}\right)$ & $a_{i-1}(\mathrm{~mm})$ & $d_{i}(\mathrm{~mm})$ & $\theta_{i}\left({ }^{\circ}\right)$ \\
\hline 1 & $-\mathrm{pi} / 2$ & 100 & 260 & 0 \\
2 & 0 & 290 & 0 & $\mathrm{pi} / 2$ \\
3 & $\mathrm{pi} / 2$ & -121 & 0 & 0 \\
4 & $-\mathrm{pi} / 2$ & 0 & 310 & 0 \\
5 & $\mathrm{pi} / 2$ & -112.5 & 0 & $-\mathrm{pi} / 2$ \\
6 & 0 & 0 & 0 & 0 \\
\hline
\end{tabular}

\section{Kinematic equations of the robot}

According to DH method, when $i=1,2,3,4,5,6$, the homogeneous transformation matrix is Eq. (1):

$T_{i-1, i}=\operatorname{Rot}\left(z_{i-1}, \theta_{i}\right) \operatorname{Trans}\left(0,0, d_{i}\right) \operatorname{Trans}\left(a_{i}, 0,0\right) \operatorname{Rot}\left(x_{i}, \alpha_{i}\right)$.

Expand the Eq. (1), get the Eq. (2):

$$
T_{i-1, i}=\left[\begin{array}{cccc}
\cos \theta_{i} & -\sin \theta_{i} \cos \alpha_{i} & \sin \theta_{i} \sin \alpha_{i} & a_{i} \cos \theta_{i} \\
\sin \theta_{i} & \cos \theta_{i} \cos \alpha_{i} & -\cos \theta_{i} \sin \alpha_{i} & a_{i} \sin \theta_{i} \\
0 & \sin \alpha_{i} & \cos \alpha_{i} & d_{i} \\
0 & 0 & 0 & 1
\end{array}\right] .
$$

The robot's kinematic equation is shown as Eq. (3):

$T_{0,6}=T_{0,1} \cdot T_{1,2} \cdot T_{2,3} \cdot T_{3,4} \cdot T_{4,5} \cdot T_{5,6} \cdot$

\section{The kinematic modeling simulation based on Matlab}

Matlab is a kind of matrix arithmetic programming tool with visual and powerful function. It has been widely used in numerical calculation, product development, industrial research and scientific calculation.

In this paper, Robotics toolbox (its interface is shown in Fig. 3) was used to carry on the kinematic simulation, and the graphic model of the 6-DOF industrial robot is shown in Fig. 4. 


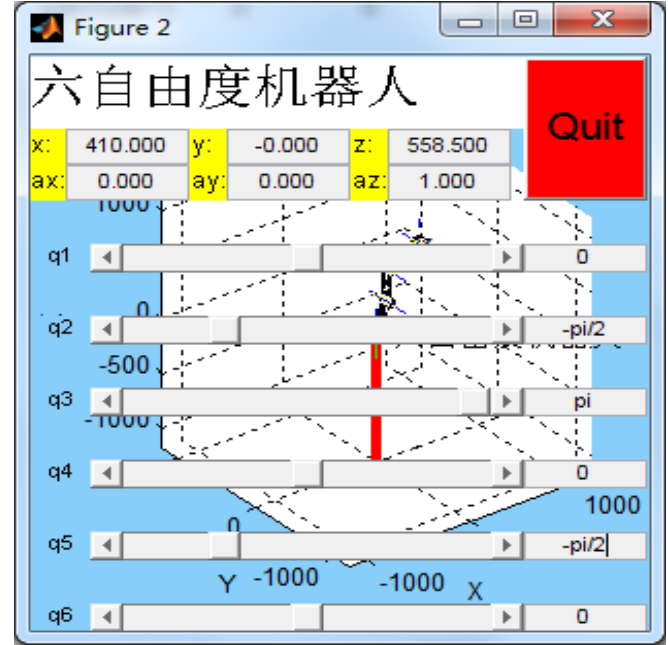

Fig. 3 The interface of the robotics toolbox

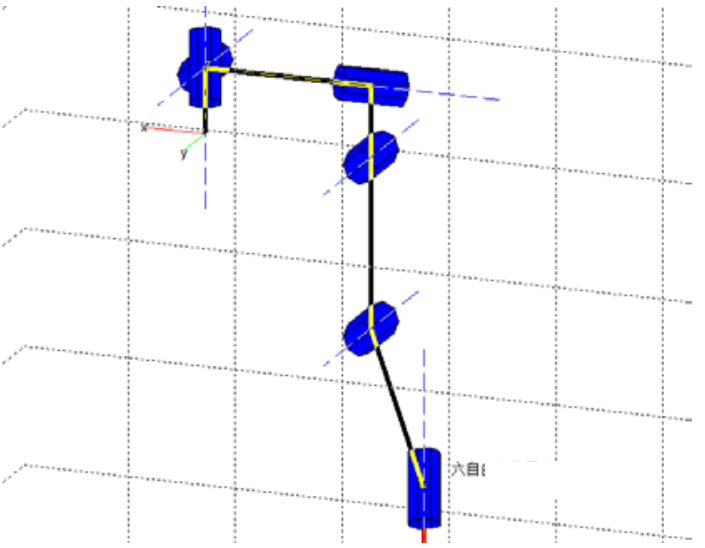

Fig. 4 The graphic model of the robot

The control interface is shown in Fig. 3. As $q_{1}-q_{6}$ is corresponding to the input joint angles $\theta_{1}-\theta_{6}$, the values of input joint angle can be changed by dragging the slider of $q_{1}-q_{6}$ accordingly. The established kinematic model can satisfy the kinematic model of the AACMM if the changes of joint angle are consistent with the graphic simulation in the Matlab.

By comparison, the calculation result based on the kinematic model and the graphic model of the robot were matched.

\section{Experiment verification}

Each joint of the experimental robots is random rotating and the same angle is joined in the simulation model, the comparison of the result between the simulation and the experiment in the zero position is shown in Fig. 5. We selected ten points to be tested, including that the first point is the robot's zero position, the other points are random positions. The coordinates of the experiment robot can be extracted directly by the robot controller, and the coordinates of the robot controller are defined as the true values. The coordinates of the robot's end-effector are defined as the simulation values in the simulation model. The correctness of the established kinematics model can be judged by analyzing the calculated measuring coordinates and actual coordinates gathered by data acquisition software randomly. The joint angles save as integers and the values save as a decimal.

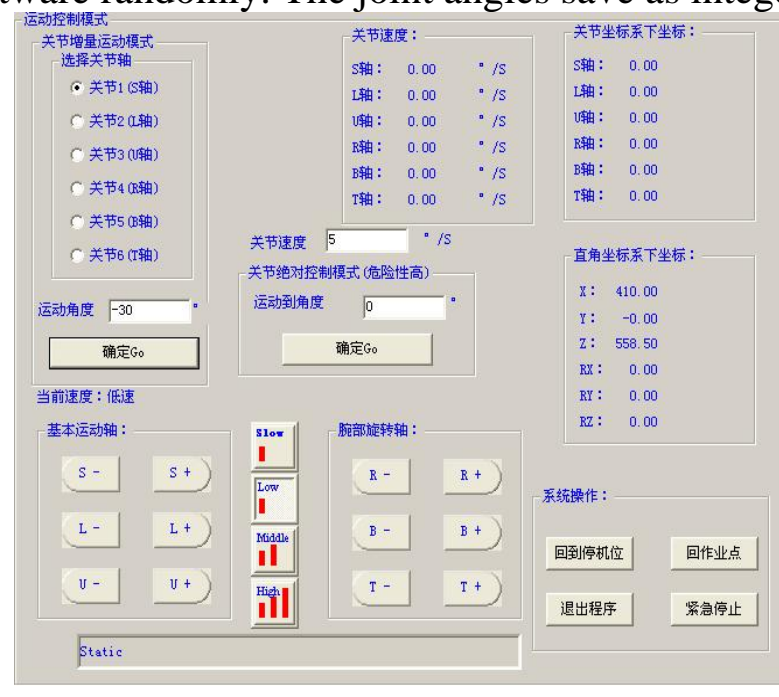

(a) The experiment display of the robot

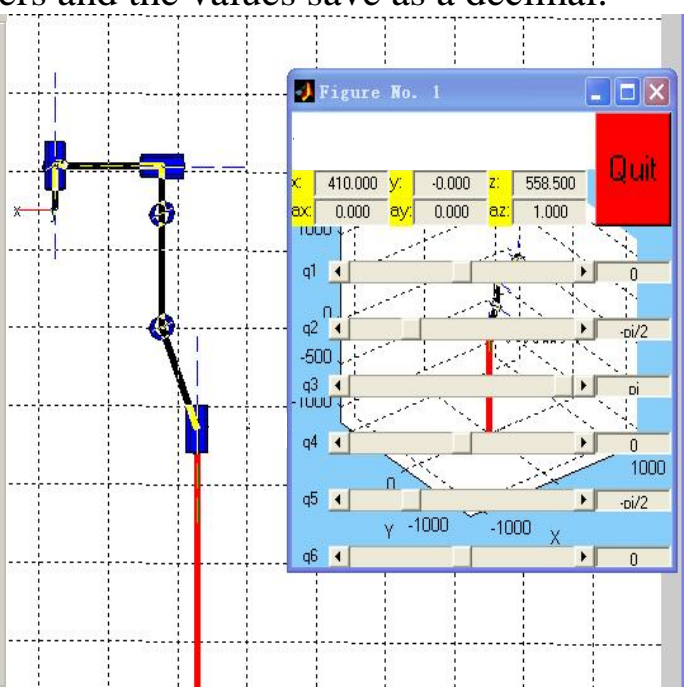

(b) The graphic simulation of the robot

Fig. 5 The comparison of the result between the simulation and the experiment in the zero position 
According to the analysis of data in Table 2, the error between the entity robot coordinate and the simulation robot coordinates is same, shown that the kinematics equation and model are reliable, as shown in Table 2.

Table 2 The results of the robot coordinate comparison

\begin{tabular}{cccccccc}
\hline & & \multicolumn{3}{c}{ The reference coordinates } & \multicolumn{4}{c}{ The coordinates of the calculation } \\
NO. & $\theta(\theta 1, \theta 2, \theta 3, \theta 4, \theta 5, \theta 6)\left({ }^{\circ}\right)$ & $\mathrm{X}$ & $\mathrm{Y}$ & $\mathrm{Z}$ & $\mathrm{X}$ & $\mathrm{Y}$ & $\mathrm{Z}$ \\
\hline 1 & $(0,0,0,0,0,0)$ & 410.00 & 0.00 & 558.50 & 410.00 & 0.00 & 558.50 \\
2 & $(30,30,0,30,-90,-10)$ & 581.45 & 335.70 & 404.69 & 581.44 & 335.70 & 404.69 \\
3 & $(-10,-20,10,10,30,0)$ & 201.94 & -52.79 & 681.08 & 201.94 & -52.79 & 681.08 \\
4 & $(30,-30,25,-50,10,0)$ & 46.79 & 125.12 & 809.64 & 46.79 & 125.12 & 809.64 \\
5 & $(20,10,30,0,0,0)$ & 412.30 & 150.06 & 659.61 & 412.30 & 150.06 & 659.61 \\
6 & $(-30,16,-32,-20,30,-15)$ & 338.49 & -156.95 & 369.90 & 338.49 & -156.95 & 369.90 \\
7 & $(-50,45,30,20,7,-17)$ & 353.47 & -480.66 & 403.90 & 353.47 & -480.66 & 403.90 \\
8 & $(50,32,19,-21,4,13)$ & 385.46 & -396.80 & 453.78 & 385.46 & -396.80 & 453.78 \\
9 & $(-29,16,-25,0,-15,35)$ & 388.30 & -215.24 & 325.59 & 388.30 & -215.24 & 325.59 \\
10 & $(50,-10,20,18,-18,30)$ & 242.97 & 238.13 & 734.64 & 242.97 & 238.13 & 734.64 \\
\hline
\end{tabular}

\section{Conclusion}

The kinematic modeling method of the industrial robot was studied in this paper. DH method was chosen to establish the kinematic model of a 6-DOF robot. Then the matrix from the joint space to the working space of the robot was achieved through homogeneous transformation. Simulation calculation and experiments results show that the kinematic model is correct.

\section{Acknowledgement}

This research was financially supported by the National Natural Science Foundation of China (Grant No. 51465027).

\section{References}

[1] W. Bu, Z. Liu, and J. Tan. "Industrial Robot Layout Based on Operation Sequence Optimisation." International Journal of Production Research 47, no. 15 (2009): 4125-45.

[2] G. Gao, J. Lu, and J. Zhou. "Kinematic Modeling for a 6-Dof Industrial Robot." Paper presented at the 2012 International Conference on Mechatronic Systems and Materials Application, Qingdao, China, September 8 - 9, 2012.

[3] Y. Gong and Y. Xu. "The Study on Mechanical Design Based on Solidworks and Simulink." International Journal of Advancements in Computing Technology 4, no. 21 (2014): 430-37.

[4] J. Santolaria and G. Manuel. "Uncertainty Estimation in Robot Kinematic Calibration." Robotics and Computer-Integrated Manufacturing 29, no. 2 (Apr 2013): 370-84.

[5] L. Wang, X. Chen, Q. Huang, X. Wang, and P. Guo. "Error Analysis of Multi-Legged Walking Robots Based on Forward Kinematics." Yalong Bay, Sanya, China, 2008. 